\title{
Surface and implantation effects on $p-n$ junctions
}

\author{
S E Schachamt and E Finkman \\ Department of Electrical Engineering and Solid State Institute, Technion-israel \\ Institute of Technology. Haifa 32000, Israel
}

Received in accepted form 18 September 1989

\begin{abstract}
The contribution of the graded region of implanted $p-n$ junctions is analysed using an exponential profile. Though previously neglected, we have recently shown that this contribution to the saturation current of HgCdTe diodes is significant. Assuming a dominant Auger recombination, an analytical solution to the continuity equation is obtained. An expression for the current generated by the graded region is presented for both ohmic and reflecting boundary conditions. A revised condition for a 'wide' region is derived. When the region is 'narrow'. the current differs drastically from that of the zero-gradient case. The effects of the junction depth and the substrate and surface concentrations on the current are investigated. It is shown that the reverse current does not saturate.
\end{abstract}

\section{Introduction}

The structure of $p-n$ junctions made by microcircuit fabrication techniques is graded. When generating such junctions either by diffusion or by implantation. the carrier concentration on the diffused implanted region is not constant. This structure has several implications as to the performance and mathematical analysis of such diodes. The non-constant dopant profile gives rise to an electric field throughout the region. which directs the minority carriers back into the junction. The carrier diffusion current is modified and a drift current component is added. The excess carrier lifetime varies according to the local carrier concentration. thus affecting the junction characteristics.

Most methods for analysing I-I characteristics of $p-n$ junctions are based on the depletion approximation. This approximation renders exceilent results for an abrupt junction. Howerer. the mathematical analysis of graded junctions cannut rely on the abrupt junction model. As a first-term expansion. the linearly graded junction [1, 2] suffers from serious basic drawbacks and its accuracy is questionable. An exponential carrier profile is a very attractive alternative [3.5]. Accurate numerical calculations for both gaussian and erfe doping gradients can be closely approximated by an exponential function [4]. The latfer hals several convenient features: in many cases it can be solved analytically, the electric field generated by such a carrier profile is constant, and mathematically this profile is consistent with the depletion approximation [3].

+ Mailing address: NASA Lewis Resedrch Center. m. 545 , Clevelind. OH $4+135$, LSA.

0268-1242/90/030S41-04 \$03.50 C 1990 IOP Publishing Ltd
The narrow-gap semiconducwer He: . Cd Te is the leading materiat for making intrared photodoleciors. Depending an the somposition ratio $x$ the sarious atmosplecrie windows can be colered. Imaging arratyate

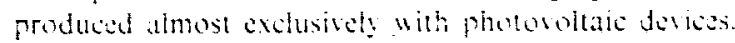
There diodes are mosty implemented h: ion implantatifon. usually resulting in an n layer on a p-iype subutrat [6. 7]. Recent reports have shown that diftusion and heat tratament of an HgCJTe substrate can turn the top layer into it p-region. thus producing a p-on-n junction [s. 9].

The general approach to the analysis of the performance of $H g_{1}, C d_{*} T_{L}$ diodes touk into consideration the substratc only. employing a one-side diffusion model for abrupt junctions. This approach was based on the assumption that the graded region does not contribute to the current [10.11]. Recently wa have proven experimentally that there is a simificum current component generaled by the implinted $n^{-}$graded region of $\mathrm{Hg}-\mathrm{Cd}_{\mathrm{b}} \mathrm{T}$ diodes [12]. Depending on temperature, the ratio beween the eurrent produced by the graded region and that generated by the substrate is 0.53 . Therefore we proceed to investigalte this contribution.

The analysis of the elfects of inglantation conditions and surlace trealment on the performance of $\mathrm{Hg}_{\mathrm{b}_{-}-x} \mathrm{Cd_{x }} \mathrm{Te}_{\mathrm{e}}$ photodiodes is the subject of this paper. The implantation parameters determine the carrier gradient and the junction depth. Surface treatment, as manifested by boundary conditions, is an essential part of this analysis. It is well known that for abrupt junctions. reflecting boundary conditions render photodiodes with superior performance. i.e. higher $R_{1} t$ and lower saturation current [10]. Since the electric field present outside the depletion region directs the minority carriers away 
from the surface, this property has to be investigated for a graded region.

\section{Current generated by graded region}

The current generated by the graded region is derived from the excess carrier distribution. This distribution can be obtained from the continuity equation, taking into account the electric field present outside the depletion layer, and introducing the concentration dependence of the various parameters. Since the graded region is characterised by a high concentration of carriers, we assume the lifetime is dominated by an Auger process. Let us assume that the graded region is $\mathrm{n}$-type over a $\mathrm{p}$ substrate (as is usually the case in $\mathrm{HgCdTe}$ ). The carrier profile in the graded region is approximated by an exponential function

$$
N(x)=N_{0} \exp \left(-\eta x / x_{j}\right)
$$

where $N_{0}$ is the surface concentration, $x_{j}$ is the junction depth and $\eta=\ln \left(N_{0} / N_{0}\right)$ wherc $N_{0}$ is the substrate concentration. This distribution results in a constant electric field $E=k T \eta / q x_{j}$ throughout the graded region. The resulting continuity equation can be solved analytically [13]. If the lifetime is indeed dominated by Auger recombination. a hyperbolic function is obtained for the excess carrier distribution. The current contribution of the graded region hals two components: the diffusion term, obtained by taking the derivative of the excess hole distribution at the edge of the depletion region, $x_{n}$, and a drift term due to the constant electric field.

The solution of the continuity equation depends on the boundary conditions at the surface. For ohmic contacts, i.e. with no excess carriers at $x=0$, the saturation current generated by the graded region is given by

$$
J_{0 n t}(\text { ohmic })=J_{x} \operatorname{coth}\left(\frac{x_{1}}{\eta}\left(L(0)^{-1}-L\left(x_{n}\right)^{-1}\right)\right) .
$$

The diffusion length $L$ depends on position through the lifetime dependence on concentration: $L(0)$ is its value at the surface, while $L\left(x_{n}\right)$ is the value at the edge of the depletion layer. Here $J_{x}$ is the "classical' saturation current for a 'wide' region. i.e. $x_{\mathrm{j}} \geqslant L$, of an abrupt junction with a constant carrier concentration:

$$
J_{x}=q \frac{D_{p} \bar{p}\left(x_{n}\right)}{L\left(x_{n}\right)}
$$

It is important to note that for the case of a dominant Auger recombination, $J_{x}$ is independent of location and is a material parameter, since the equilibrium minority carrier concentration at the edge of the depletion region. $\bar{p}\left(x_{n}\right)$, is equal to $n_{i}^{2} / N\left(x_{n}\right)$, and since the Auger lifetime depends inversely on the square of majority concentration. Usually, owing to the steep slope of the carrier profile in the graded region. $L\left(x_{n}\right) \gg L(0)$; thus $J_{0 n}($ ohmic $) \approx J_{\infty} \operatorname{coth}\left(x_{j}, \eta L(0)\right)$. This approximation is valid only if the width of the neutral region, $x_{n}$, is not much smaller than $x_{j}$ (not close to punch-through). Under these approximations, the exact value of $x_{n}$ is immaterial, even though this width may be smaller than the diffusion length. For a graded junction we have to revise the condition for a 'wide' region to $x_{j} \gg \eta L(0)$ : then $J_{\text {in }}$ (ohmic) approaches $J_{x}$. However, when the region is 'narrow', we obtain a drastic reduction in $J_{\text {on }}$ by a factor of $\eta w_{n} L(0) / x_{j} L\left(x_{n}\right)=\eta w_{n} N\left(x_{n}\right), x_{j} x_{n}$ over the abrupt case with a constant concentration $v_{d}$ equal to $N\left(x_{n}\right) ; w_{n}$ is the width of the neutral region for the abrupt junction. Only when the constant concentration of an abrupt junction is of the order of the surface concentration are the currents comparable. When $V_{d}=N_{0}$, the factor is $\eta w_{n}<x_{j}$.

It should be pointed out that although the final formulation of the saturation current for the graded junction is very similar to that of the abrupt one, this may be misleading. The local carrier concentration is drastically different. The electric field tends to repel the minority carriers back to the junction; thus the carriers have to redistribute in order to comply with the same boundary conditions. The drift component of the current always opposes the diffusion component.

The dual boundary condition is that of a perfectly reflecting surface. Solving the continuity equation with the appropriate limits, and obtaining both the diffusion and the drift terms at the edge of the depletion region. the contribution of the graded region to the saturation current is found to be

$$
J_{i n n}(\text { reflecting })=j, \operatorname{lanh}\left(\frac{x_{j}}{\eta}\left(L(0)^{-1}-L\left(x_{n}\right)^{-1}\right)\right) .
$$

For a steep enough profile. the current can be approx. mated by $J_{o n}$ (reflecting) $=J_{x} \tanh (x, \eta L(0))$. It the graded region is wide according to the rovised definition. the saturation current approaches the same asymptotic value - that of a wide abrupt junction. For a narrow region, the ratio between the current of a graded structure and that of an abrupt junction is the inverse of the ratio derived for ohmic contact. It should be noted thit the current for the reflecting boundary is always smaller than $J_{x}$, while the current for the ohmic contaet is always larger than this value. Thus $J_{0 n}$ (ohmic) is ahals larger than $J_{0 \mathrm{n}}$ (reflecting), just as in the case of an abrupt junction.

Next let us analyse the dependence of the saturation currents on the various device parameters. The effect of the junction depth $x_{j}$ on $J_{0}$ is similar to that for the case of a constant-concentration region [10], i.e. the shallower the junction, the larger is the current for ohmic contact and the opposite for reflecting contact. Again, though. the physical processes involved are much more involved for the graded junction. While for an abrupt junction the only effect of bringing the junction closer to the surface is the increased excess carrier gradient for ohmic contact, thus increasing their diffusion, in a graded junction the shallower junction results also in a larger electric field which opposes the current in the case of ohmic contacts. For reflecting conditions, since no net flow should reach the boundary, the change in the electric field just described must be compensated by an appropriate redistribution of excess carricrs. These results are demonstrated 
in figure 1, where we have plotted the saturation current as a function of the normalised depth. The exponential profile is compared with two abrupt junctions whose constant concentrations are $N_{\mathrm{d}}=N_{\mathrm{o}}\left(=5 \times 10^{17}\right)$ and $N_{\mathrm{d}}=N\left(x_{\mathrm{n}}\right)$. The problem is that $x_{\mathrm{n}}$ and $N\left(x_{\mathrm{n}}\right)$ change with changing $x_{\mathrm{j}}$. We took $N_{\mathrm{d}}=10^{17} \mathrm{~cm}^{-3}$, which is a typical value for the smaller $x_{j} ; x_{n}$ was derived from a recent work by Pimbley [3]. All the calculations were performed for $\mathrm{Hg}_{1-x} \mathrm{Cd}_{x} \mathrm{Te}$ diodes with composition $x=0.21$, i.e. with $77 \mathrm{~K}$ band gap which covers the second atmospheric window.

Applying this analysis, other important conclusions can be drawn, such as the effect of the surface concentration on the current. Increasing the surface concentration (maintaining $N_{\mathrm{a}}$ and $x_{\mathrm{j}}$ constant) increases the gradient and the electric field while reducing the excess carrier lifetime throughout the graded region. Mathematically, two factors in the argument of the hyperbolic functions are affected: $L(0)$ is inversely proportional to $N_{0}$, while $\eta$ is proportional to its logarithm. Thus increasing the surface concentration reduces $J_{0}$ for ohmic boundary conditions and increases it for reflecting conditions. These effects are shown in figure 2.

A similar analysis can be applied to investigate the effects of substrate concentration on the performance of the graded junction. Increasing $N$, while maintaining a constant $N_{0}$ and $x_{j}$ is achieved by decreasing the profile gradient, reducing both the electric field and the excess carrier lifetime. The consequence is a smaller $\eta$; thus the current decreases for ohmic contacts and increases for

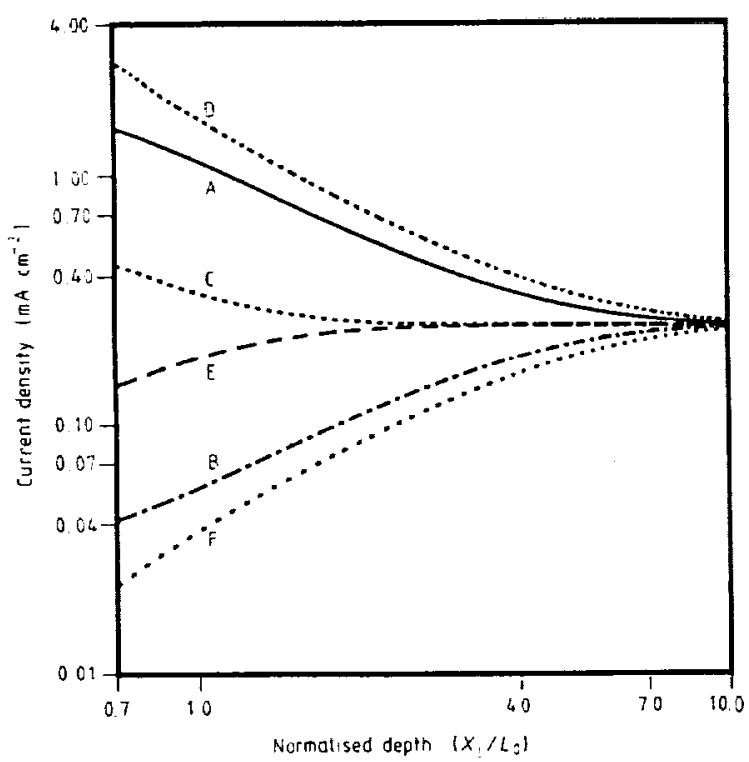

Figure 1. Saturation current generated by an n-region against normalised junction depth, for an exponential profile and for abrupt junctions with $N_{d}=5 \times 10^{17}=N_{0}$ and $N_{d}=10^{17} \approx N\left(x_{n}\right)$, for ohmic and reflecting boundary conditions $\left(\mathrm{Hg}_{1}, \mathrm{Cd}_{x} \mathrm{Te}, x=0.21, N_{\mathrm{a}}=10^{18}\right): \mathrm{A}$, graded ohmic; $B$, graded reflecting; C, constant ohmic, $N_{d}=N_{0} ; E$ constant reflecting, $N_{\mathrm{d}}=N_{\mathrm{o}}$ : D. constant ohmic, $N_{\mathrm{o}}=N\left(x_{n}\right)$ : $F$, constant reflecting, $N_{a}=N\left(x_{n}\right)$.

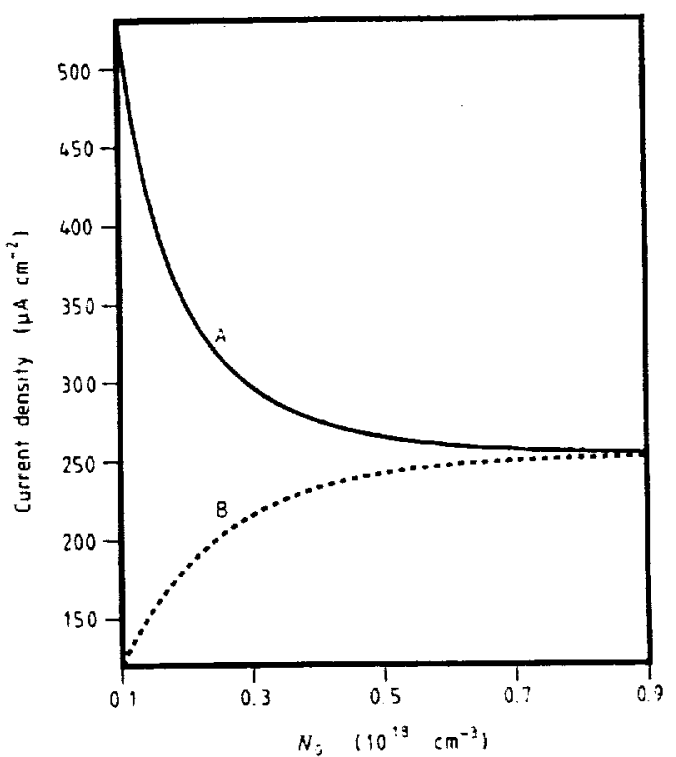

Figure 2. Saturation current generated by an n-region of a graded junction as a function of surface concentration for (A) ohmic and (B) reflecting boundary conditions $\left(\mathrm{Hg}_{1}{ }_{\mathrm{x}} \mathrm{Cd}_{\mathrm{x}} \mathrm{Te}, \mathrm{x}=0.21, \mathrm{~N}_{\mathrm{a}}=10^{16}, \mathrm{x}_{1}=0.4 \mu \mathrm{m} . \mathrm{T}=77 \mathrm{~K}\right.$, $V=-0.1 \mathrm{~V}$; Auger recombination).

reflecting surfaces. As $N$, approaches $N_{n} . L\left(x_{n}\right)$ approaches $L(0)$. This effect is enhanced by the widening of the depletion region. The combined result is that the saturation current does not reach $J_{2}$. as shown in figure 3. For the last two figures we assumed a typical reverse bias of $0.1 \mathrm{~V}$. The exact bias has little implication on this analysis.

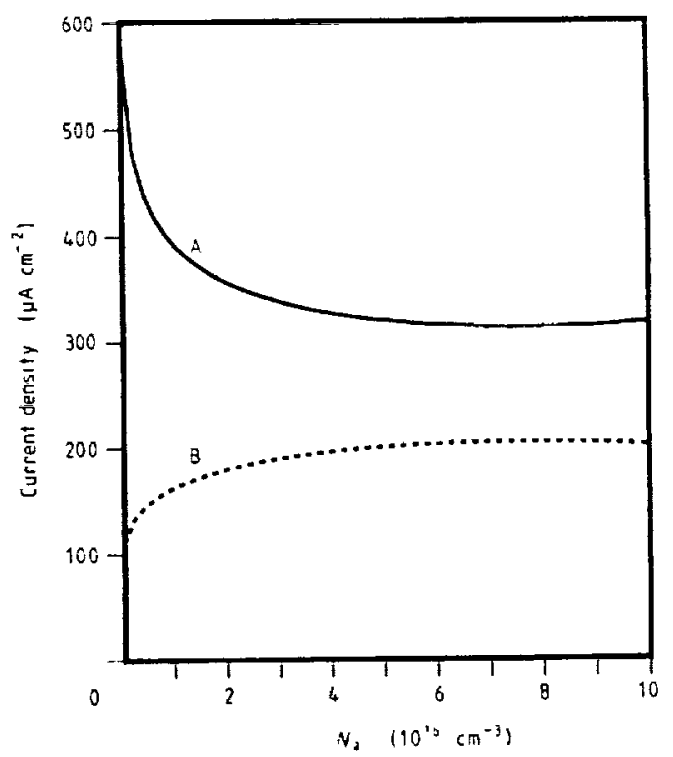

Figure 3. Saturation current generated by an n-region of a graded junction as a function of substrate concentration for (A) ohmic and $(B)$ reflecting boundary conditions $\left(\mathrm{Hg}_{1-x} \mathrm{Cd}_{x} \mathrm{Te}, \mathrm{x}=0.21, \mathrm{~N}_{0}=4 \times 10^{17}, \mathrm{x}_{1}=0.2 \mu \mathrm{m}\right.$, $T=77^{*} \mathrm{~K}, \mathrm{~V}=-0.1 \mathrm{~V}$; Auger recombination) 
Another interesting effect is the dependence of the saturation current on the reverse bias. Even though we assume that the current is not governed by generation and recombination nor by tunnelling, the reverse current may not approach a saturation value. This effect, shown in figurc 4 , is a consequence of $x_{n}$ moving towards the surface with increasing reverse bias.

\section{Conclusions}

The current generated by a graded region of a $p-n$ junction difers considerably from that generated by a region of constant carrier concentration. The exact expression for the current component depends on the recombination mechanism, surface treatment, exact carrier profile and substrate concentration. The illustrations provided in this paper are specific to $\mathrm{Hg}_{\mathrm{t}} \mathrm{Cd}_{x} \mathrm{Te}$.

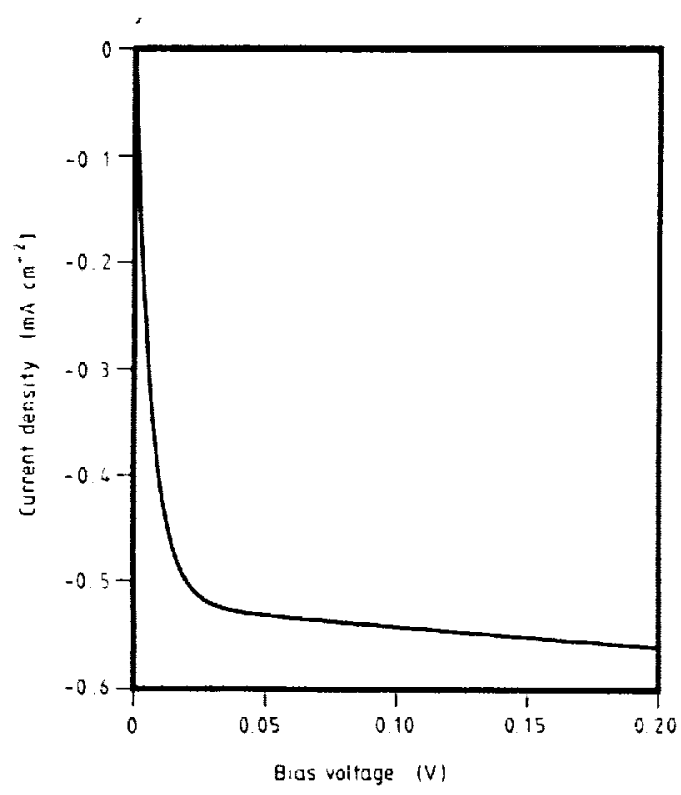

Figure 4. $1-V$ characteristic for reverse bias showing the current generated by an n-region of a graded $\mathrm{Hg}_{1}{ }_{x} \mathrm{Cd}_{x} \mathrm{Te}$ diode $\left(x=0.21, N_{*}=10^{16}, N_{0}=5 \times 10^{17}, x_{1}=0.1 \mu \mathrm{m}\right.$, $T=77 \mathrm{~K}$; ohmic contact). Note that the reverse current does not saturate. dominated by the Auger 1 recombination process, with lifetime inversely proportional to the square of the carrier concentration. As the graded n-type region may very likely be degenerated, the recombination may have a different power law. In this case, as well as in cases dominated by radiative or Shockley Read-type recombination, the current is expressed as a combination of modified Bessel functions [13]. Still, the simplicity of our solution makes it a very attractive tool for investigation of the basic processes involved and how they are influenced by selection of the proper parameters. Finally. the new p-on-n junctions may present a structure for which the analysis is exact since the p-type material does not become degenerate till much higher concentrations. The expressions for the current are obtained merely by replacing the Auger 1 process by an Auger 7 .

\section{Acknowledgment}

We wish to thank Dr Y L Juravel for his interest in the early stages of this work.

\section{Relerences}

[1] e.g. Sze S M 1981 Physics of Semiconductor Detices (New York: Wiley)

[2] van Opdorp C and it Hoolt G W 1984 . Solid Stare Elextrom. $27 \geq 61$.

[3] Pimbley J M 1988 IEEE Trans. Electron Derices ED-35 195762

[4] Ghandhi S K 1968 The Theory and Pratice of Wicrodederonics (New York: Wilcy)

[5] Chawla B R and Gummel H $\mathrm{K}$ 19-1 IEEE Trams. Electron Deteres ED-18 17895

[6] Marine I and Motte C 1973 thpl. Phys. Letr. $23+50 . ?$

[7] Kolodny A and Kidron I 1980 JEEE Truns. Elestrm Derices ED-27 $37+3$

[8] Arias J M, Shin S H. Pasko J G. DeWames R E and Gertner E R 1989 J. Appl Phys 65174753

[9] Destefanis G L $1988 \mathrm{~J}$. Crost. Griw/h 86 700-22

[10] Reine $M B$, Sood $A K$ and Tredwell $T J 1981$ Semiconductors and Semimeralis vol. 18 . ed. $\mathrm{R} \mathrm{K}$ Willardson and A C Beer (New York: Academic) pp $201-311$

[11] Tredwell T J and Long D 1977 Find Tt'th. R:p.. NAS.t Contrace $1.159-1+180$

[12] Schacham S E and Finkman E $1989 \mathrm{~J}$ like Sit. Teithol. A 7 387-90

[13] Schacham S E and Finkman E. to be published 